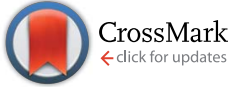

Cite this: RSC Adv., 2017, 7, 3287

Received 9th November 2016 Accepted 8th December 2016

DOI: 10.1039/c6ra26556a

www.rsc.org/advances

\title{
The dripping-to-jetting transition in a co-axial flow of aqueous two-phase systems with low interfacial tension $\uparrow$
}

\author{
Sze Yi Mak, ${ }^{\text {ab }}$ Youchuang Chao ${ }^{\mathrm{ab}}$ and Ho Cheung Shum ${ }^{\text {ab }}$
}

The dripping-to-jetting transition of co-axial flow with high interfacial tension has been extensively studied; however, little is known about this with low interfacial tension. We use an aqueous two-phase system as a model to study the transition at low interfacial tension $\left(<1 \mathrm{mN} \mathrm{m}^{-1}\right)$. We identify a low critical jet velocity at which the transition occurs $\left(V<1 \mathrm{~m} \mathrm{~s}^{-1}\right)$ when compared with the velocity in systems with high interfacial tension $\left(V>1 \mathrm{~m} \mathrm{~s}^{-1}\right)$. The significant reduction in the critical jet velocity alters the relative importance of the inertial force $\left(\propto V^{2}\right)$ and the viscous force $(\propto V)$. Counter-intuitively, even for systems with relatively low viscosities, the transition is no longer inertially-dominated. Therefore, we propose that all downstream forces, including inertial and viscous forces, contribute significantly in opposing the interfacial force. This comprehensive force balance accurately characterizes the dripping-to-jetting transition for a wide range of viscosities and low interfacial tension. The validity of this force balance is confirmed by the agreement in the interfacial tension values estimated by inputting the operating parameters of the flow into the force balance and that obtained by a commercial spinning drop tensiometer.

\section{Introduction}

Co-axial flow of two immiscible fluids exhibits dripping and jetting phenomena, which refer to the formation of drops and a jet at the nozzle respectively. ${ }^{1-3}$ The dynamics of the drippingto-jetting transition in co-axial flow is of fundamental importance in industrial applications that involve droplets. ${ }^{4-6}$ Oilwater systems with a relatively high interfacial tension $(\gamma=0.1-$ $40 \mathrm{mN} \mathrm{m}^{-1}$ ) are the main options to study the transition. Recently, some novel systems with a low interfacial tension, such as aqueous two-phase systems (ATPSs), are used to generate droplets and have great potential for applications in making bio- and cyto-compatible emulsion droplets. ${ }^{7-13}$ However, dripping is difficult to achieve spontaneously in aqueous two-phase flows, evidenced by the reliance on mechanical ${ }^{8,14}$ or electrical ${ }^{7,15}$ actuations to control the breakup of water-water jets, if not passively generated by hydrostatic flows. ${ }^{16}$ As a result, the dynamics of the dripping-to-jetting transition in these systems is rarely studied, despite lots of

${ }^{a}$ Department of Mechanical Engineering, The University of Hong Kong, Pokfulam Road, Hong Kong, China. E-mail: ashum@hku.hk

${ }^{b} H K U$-Shenzhen Institute of Research and Innovation (HKU-SIRI), Shenzhen, Guangdong, China

$\dagger$ Electronic supplementary information (ESI) available: Preparation of ATPSs, compositions and fluid properties of ATPSs, movies for dripping and jetting morphologies, and the figure of $\mathrm{We}_{\mathrm{i}}$ against $\mathrm{Ca}_{\mathrm{o}}$ for ATPSs. See DOI: 10.1039/c6ra26556a works reported for higher interfacial tension $(\gamma=0.1-40 \mathrm{mN}$ $\mathrm{m}^{-1}$ ) using oil-water systems. ${ }^{17-21}$

It is generally accepted that the dripping-to-jetting transition is typically achieved by the dominance of any of the downstream forces over the interfacial force. Strong inertial force of the inner phase $\left(F_{\text {inertial }} \sim \rho_{\mathrm{i}} L_{\mathrm{i}}{ }^{2} V_{\mathrm{i}}^{2}\right)$ and viscous forces of the inner and outer phases $\left(F_{\text {viscous,i }} \sim \mu_{\mathrm{i}} L_{\mathrm{i}} V_{\mathrm{i}}\right.$ and $\left.F_{\text {viscous,o }} \sim \mu_{\mathrm{o}} L_{\mathrm{i}} V_{\mathrm{o}}\right)$ can be achieved by a high velocities or high viscosities respectively. ${ }^{17,20,22,23}$ Inertial-dominated breakup is commonly characterized by the Weber number of the inner phase approaching unity $\left(\mathrm{We}_{\mathrm{i}} \sim 1\right)$, without considering the viscous force of the inner phase. Viscous-dominated breakup occurs only if the jet phase is highly viscous, such as in the case of glycerol or sucrose with viscosity on the order of 1-10 Pa s. ${ }^{24-26}$ However, waterwater interfaces typically possess low interfacial tension ranging from $\mathrm{O}\left(10^{-3}\right)$ to $\mathrm{O}(1) \mathrm{mN} \mathrm{m}^{-1} \cdot{ }^{27}$ The lowering of interfacial tension by a thousand fold leads to a very small driving force for jet breakup, as well as a slow breakup dynamics. ${ }^{15}$ Therefore, to reveal the details of the dripping-to-jetting transition in low interfacial tension systems, a systematical view of the balanced forces is necessary.

In this work, we use aqueous two-phase systems (ATPSs) as a platform to study the dripping-to-jetting transition in the low interfacial tension system. We find that the jet velocity at the transition reduces by 1000 -fold when the interfacial tension is low and viscosity is low. The relative importance of the inertial and viscous forces at the dripping-to-jetting transition is completely altered. Therefore, we propose a modified 
characterization of the dripping-to-jetting transition which is applicable even when the interfacial tension is low. Our results elucidate the underlying mechanism for the dripping-to-jetting transition in low-interfacial-tension systems, and thus provide a more representative phase diagram for the dripping-to-jetting transition in two-phase co-axial flow. The understanding of the roles of different hydrodynamic forces in the breakup of waterwater jets not only has implications to controlled generation of bio- and cyto-compatible emulsions and particles, ${ }^{\text {,11,28-31 }}$ jets and fibers, ${ }^{\mathbf{1 0}, 14,32,33}$ but also has application to estimate low interfacial tension.

\section{Experimental}

\section{Aqueous two phase systems}

Immiscible aqueous phases are prepared by dissolving and phase-separating high concentrations of a polymer poly(ethylene glycol) MW 4000, or 8000 (10 wt\% to $23.29 \mathrm{wt} \%),{ }^{35}$ and a salt, sodium citrate $(10 \mathrm{wt} \%$ to $12 \mathrm{wt} \%)$ or sodium carbonate (6.44 $\mathrm{wt} \%$ to $10.27 \mathrm{wt} \%$ ) in deionized water (see Fig. S1 in the $\mathrm{ESI} \dagger$ for preparation of ATPSs). The interfacial tension is typically low and ranges from $0.02 \mathrm{mN} \mathrm{m}^{-1}$ to $3.2 \mathrm{mN} \mathrm{m}^{-1}$ (SITE100, KRÜSS) while the viscosity ratio varies between 0.04 and 45 ( $\mu$ VISC, RheoSense). The specific compositions and corresponding fluid properties of the ATPSs and oil-water system are listed in Table S1 in the ESI. $\dagger$

\section{Microfluidics}

A co-axial flow is formed in two aligned glass capillaries, one with a tip diameter $L_{\mathrm{i}}$ ranging from $20 \mu \mathrm{m}$ to $200 \mu \mathrm{m}$, as tapered by a micropipette puller (P-97, Sutter), inside another with a channel dimension $L_{\mathrm{o}}$ ranging from $400 \mu \mathrm{m}$ to $2 \mathrm{~mm}$. At this length scale, the Bond number Bo $\sim \Delta \rho g L_{\mathrm{i}}^{2} / \gamma$, where $g$ is the gravitational constant, is on the order of $10^{-3}$, indicating a negligible effect of gravity relative to the interfacial tension. The inner and outer phases are injected from the two inlets into the device using syringe pumps (LSP01-2A, Longer Pump) at flow rates $Q_{\mathrm{i}}=1-20 \mathrm{ml} \mathrm{h}^{-1}$ and $Q_{\mathrm{o}}=40-40 \mathrm{ml} \mathrm{h}^{-1}$, respectively (Fig. 1). Unless otherwise specified, we fix the outer flow rate and gradually increase the inner flow rate until a widening jet (Fig. 1b) is achieved.

\section{Imaging and analysis}

We capture high-speed videos of the breakup process using a high-speed camera (V9.1, Phantom; FASTCAM SA4, Photron) through an inverted microscope (AE2000, Motic). The mean velocity of the outer phase is $V_{\mathrm{o}}=4 Q_{\mathrm{o}} /\left(\pi\left(L_{\mathrm{o}}{ }^{2}-L_{\mathrm{i}}{ }^{2}\right)\right)$ or $Q_{\mathrm{o}} /\left(\left(L_{\mathrm{o}}{ }^{2}-\right.\right.$ $\left.\pi L_{\mathrm{i}}^{2} / 4\right)$ ) for circular or square cross section of the outer channel while the mean velocity of the inner phase is $V_{\mathrm{i}}=4 Q_{\mathrm{i}} /\left(\pi L_{\mathrm{i}}{ }^{2}\right)$.

\section{Results and discussion}

\section{A low critical jet velocity at the dripping-to-jetting transition}

We monitor the critical jet velocity $\left(V_{\mathrm{i}, \mathrm{t}}\right)$ at which the drippingto-jetting transition takes place for different interfacial tensions. We observe that the dripping-to-jetting transitions in low interfacial tension systems always happen at a low critical jet velocity, on the order of $\mathrm{mm} \mathrm{s}^{-1}$. However, for a typical oilwater system with a high interfacial tension of $40 \mathrm{mN} \mathrm{m}^{-1}$, this velocity is $1.7 \mathrm{~m} \mathrm{~s}^{-1}$. As the interfacial tension decreases from $2.6 \mathrm{mN} \mathrm{m}^{-1}$ to $0.012 \mathrm{mN} \mathrm{m}^{-1}$ while the viscosity of the jet phase is maintained at $1 \mathrm{mPa} s$ to $3 \mathrm{mPa}$, the critical jet velocity progressively reduces from $0.25 \mathrm{~m} \mathrm{~s}^{-1}$ to $0.002 \mathrm{~m} \mathrm{~s}^{-1}$ (Fig. 2). The extremely low critical jet velocity indicates a remarkably weak inertial force. From the literature, ${ }^{17}$ the breakup of systems with a high interfacial tension and a low viscosity of the jet phase falls into the inertial-dominated regime. In this regime, the inertial force dominates over the viscous force and is balanced by the interfacial force at the transition from dripping to jet widening, $F_{\text {inertial }} \sim \gamma L_{\mathrm{i}}$. Hence we can estimate the jet velocity at the transition by the relation:

$$
V_{\mathrm{i}, \mathrm{t}} \sim \sqrt{\frac{\gamma}{\rho_{\mathrm{i}} L_{\mathrm{i}}}}
$$

The estimation, inferred from experimental studies on systems with higher interfacial tension, only serves well to predict $V_{\mathrm{i}, \mathrm{t}}$ when the interfacial tension of the ATPS is higher than $1 \mathrm{mN} \mathrm{m}^{-1}$ but not lower (data points coincide with the solid line only when $\gamma>1 \mathrm{mN} \mathrm{m}^{-1}$ in Fig. 2). For interfacial tension lower than $1 \mathrm{mN} \mathrm{m}^{-1}$, the experimental jet velocity at the transition is lower than the extrapolated value and the deviation increases as the interfacial tension decreases further (data points deviate from the dotted line in Fig. 2).

\section{The small interfacial force of ATPS}

In fact, the critical jet velocity of the dripping-to-jetting transition is lowered by the small interfacial force of ATPSs. Specifically, for a jet ejected from a nozzle of $100 \mu \mathrm{m}$ in diameter, the downstream force required to oppose the interfacial force of an ATPS is on the order of nanonewton. Due to a tremendous reduction in interfacial force, a jet with a very small velocity possesses sufficient downstream force to overcome the minute interfacial force and causes jetting. Hence, this small critical jet velocity results in an extremely small inertial force and viscous force, all of which possibly play important roles during the dripping-to-jetting transition.

\section{The relative importance of the forces at the transition}

When the critical jet velocity is extremely low, $\left(<1 \mathrm{~m} \mathrm{~s}^{-1}\right)$, both the viscous and inertial forces of the inner phase are significantly smaller as they scale with the jet velocity $\left(F_{\text {viscous,i }} \sim \mu_{\mathrm{i}^{-}}\right.$ $\left.L_{\mathrm{i}} V_{\mathrm{i}}\right)$ and the square of it $\left(F_{\text {inertial }} \sim \rho_{\mathrm{i}} L_{\mathrm{i}}^{2} V_{\mathrm{i}}^{2}\right)$ respectively. Thus, the inertial force is reduced to a greater extent and becomes comparable to the viscous force of the inner phase, as illustrated in Fig. 3a. In the regime of low interfacial tension, the Reynolds number at the dripping-to-jetting transition $\mathrm{Re}_{\mathrm{i}, \mathrm{t}}$, which is the ratio of inertial force over viscous force, spans across three decades from $\mathrm{O}\left(10^{-3}\right)$ to $\mathrm{O}(1)$ as the viscosity ratio $\mu_{\mathrm{i}} / \mu_{\mathrm{o}}$ varies from 0.04 to 45 . This range of $\mathrm{Re}_{\mathrm{i}, \mathrm{t}}$ suggests that the transition is not inertial-dominated. Even if the viscosity of the inner phase is as low as that of water, the dripping-to-jetting 
(a)

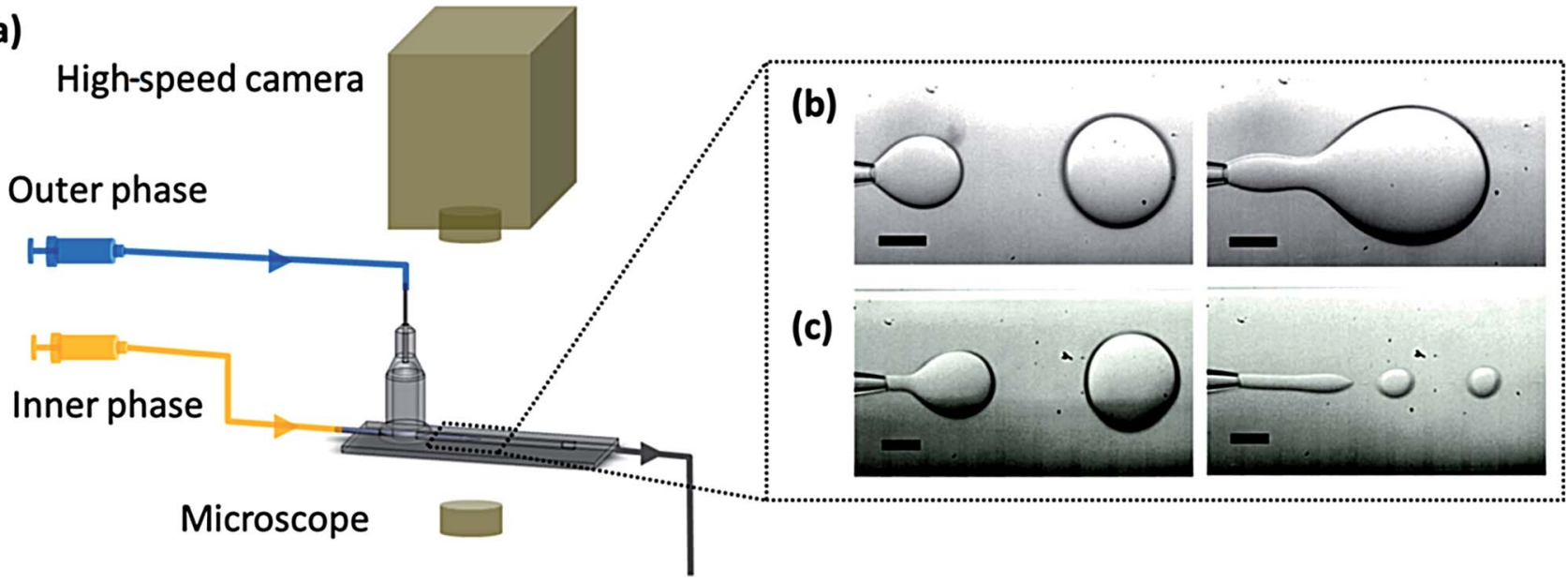

Fig. 1 (a) Schematic diagram of the experimental setup. The phase-separated aqueous phases are fed, by syringe pumps, into the glass capillary device to form a co-flow, as shown in the subset of the figure. The breakup process is observed under an optical microscope and recorded using a high speed camera. Microscopic images of two different transitions. Scale bars are $100 \mu \mathrm{m}$. (b) Transition to a widening jet by increasing the flow rate of the inner phase: $\gamma=0.26 \mathrm{mN} \mathrm{m}^{-1}, \mu_{\mathrm{i}}=1.5 \mathrm{mPa} \mathrm{s}, Q_{0}=500 \mu \mathrm{h} \mathrm{h}^{-1}$. Dripping at $Q_{\mathrm{i}}=30 \mu \mathrm{h} \mathrm{h}^{-1}$, jetting at $Q_{\mathrm{i}}=100 \mu \mathrm{h}{ }^{-1}$. (c) Transition to a narrowing jet by increasing the flow rate of the outer phase: $\gamma=0.99 \mathrm{mN} \mathrm{m}^{-1}, \mu_{\circ}=47 \mathrm{mPa} \mathrm{s}, Q_{\mathrm{i}}=100 \mu \mathrm{l} \mathrm{h}{ }^{-1}$. Dripping at $Q_{0}=1 \mathrm{ml} \mathrm{h}^{-1}$, jetting at $Q_{0}=8 \mathrm{ml} \mathrm{h}^{-1}$. See Movies S1-S4 in ESI† for the dripping and jetting morphologies.

transition is no longer sufficiently characterized by the Weber number of the inner phase (Fig. S2 $\uparrow$ ), as in the case of high interfacial tension. Neglecting the viscous force in the characterization of the dripping-to-jetting transition, as is commonly done in low viscosity systems with high interfacial tension, is not appropriate in the case of low interfacial tension. However, given the wide span of $\mathrm{Re}_{\mathrm{i}, \mathrm{t}}$ from $\mathrm{O}\left(10^{-3}\right)$ to $\mathrm{O}(1)$, it is non-trivial whether the viscous force is dominant over or comparable to the inertial force.

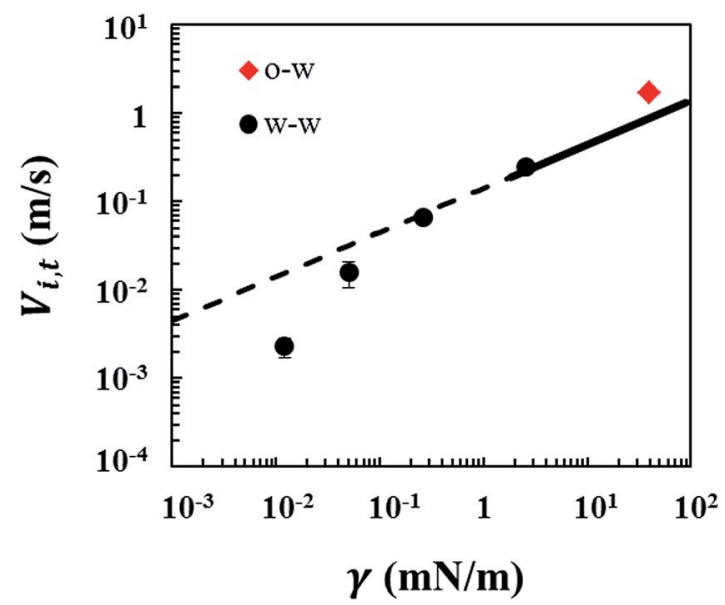

Fig. 2 Critical jet velocity at the transition from dripping to jet widening $V_{i, t}$ against interfacial tension $\gamma$ for low viscosity of the jet phase $\left(\mu_{\mathrm{i}}=1-3 \mathrm{mPa} \mathrm{s}\right)$. The diameters of the nozzle are between 40 and $80 \mu \mathrm{m}$. The solid line is $V_{i, t} \sim\left(\gamma / \rho_{i} L_{i}\right)^{0.5}$, which is obtained by balancing the inertial force with the interfacial force: $F_{\text {inertial }} \sim \gamma L_{\mathrm{i}}$. This relation is only valid for systems with interfacial tension above $1 \mathrm{mN}$ $\mathrm{m}^{-1}$ and low jet viscosity of $1 \mathrm{mPa}$. The dotted line is the relationship of $V_{i, t} \sim\left(\gamma / \rho_{i} L_{i}\right)^{0.5}$ projected to low interfacial tension. Red diamond denotes oil-water system and black circles denote water-water systems.

\section{A new characterization of the transition based on force balance}

Based on these results, we hypothesize the transition is influenced by both the inertial and viscous forces of the inner phase when the interfacial tension is low, regardless of the viscosity of the inner phase. We express the ratios of the total force contributed by the inner and outer phase over the interfacial tension force as below:

$$
\begin{gathered}
\frac{\sum F_{\mathrm{i}}}{F_{\mathrm{IFT}}}=\frac{F_{\text {inertial }}+F_{\text {viscous }, \mathrm{i}}}{F_{\mathrm{IFT}}}=\frac{\rho_{\mathrm{i}} L_{\mathrm{i}}{ }^{2} V_{\mathrm{i}}^{2}+\mu_{\mathrm{i}} L_{\mathrm{i}} V_{\mathrm{i}}}{\gamma L_{\mathrm{i}}} \\
\frac{\sum F_{\mathrm{o}}}{F_{\mathrm{IFT}}}=\frac{F_{\text {viscous }, \mathrm{o}}}{F_{\mathrm{IFT}}}=\frac{\mu_{\mathrm{o}} L_{\mathrm{i}} V_{\mathrm{o}}}{\gamma L_{\mathrm{i}}}
\end{gathered}
$$

Adopting these expressions, we propose a revised phase map that takes into account all the three downstream forces, namely the inertial force and viscous forces of the inner and outer phases, as well as the interfacial force. This new phase diagram captures the dripping-to-jetting transitions for low-interfacial tension aqueous two-phase systems with a wide range of viscosity (Fig. 3b). All the transitions of systems with different viscosity ratios collapse to the same order, where $\sum F_{\mathrm{i}} / F_{\mathrm{IFT}}$ approaches unity. The result illustrates a remarkable importance of viscous force of the jet in the breakup dynamics when the interfacial tension is low. When we increase the viscosity of the jet phase above $10 \mathrm{mPa} s$, the viscous effect is even more prominent. The transition to the jet-narrowing regime arises from increasing the speed of the continuous phase, such that the viscous force of the continuous phase overcomes the interfacial force. In general, jetting is achieved when the sum of the three downstream forces, namely the inertial force and 
(a) High interfacial tension

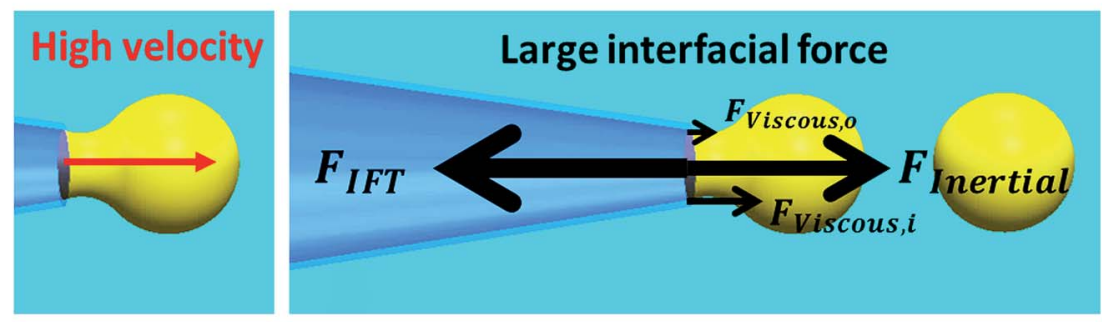

Low interfacial tension

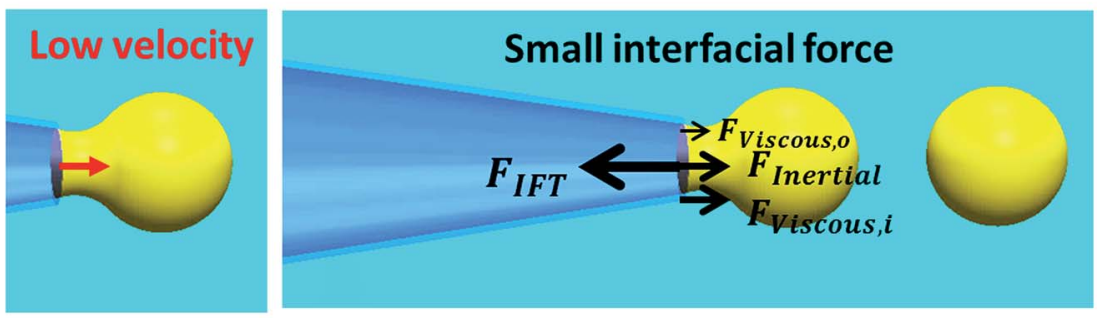

(b)

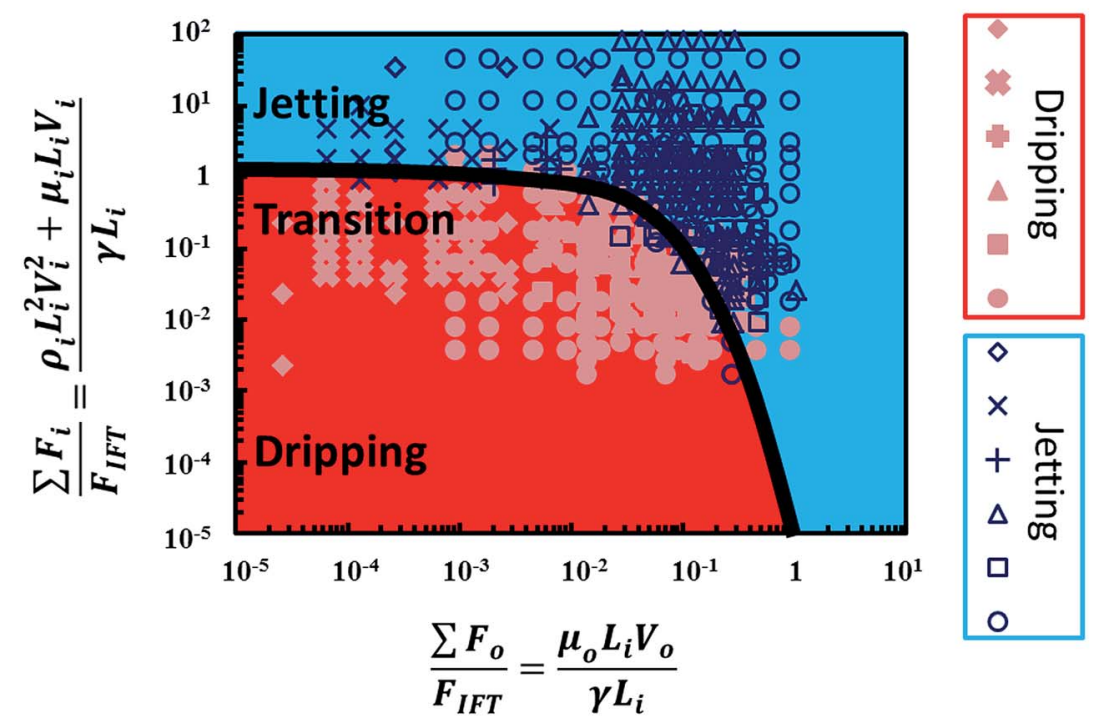

Fig. 3 (a) Schematics of the velocity and force balances at the transition. The length of arrow indicates the magnitude of the velocities and forces. Inertial force dominates when interfacial tension is high; no single force dominates when interfacial tension is low. (b) State diagram of the dripping-to-jetting transition. Circle: $\mu_{\mathrm{i}} / \mu_{\mathrm{\circ}}=0.04, \gamma=2.6 \mathrm{mN} \mathrm{m}^{-1}$. Square: $\mu_{\mathrm{i}} / \mu_{\circ}=0.05, \gamma=0.99 \mathrm{mN} \mathrm{m}^{-1}$. Triangle: $\mu_{\mathrm{i}} / \mu_{\circ}=0.09, \gamma=0.26 \mathrm{mN}$ $\mathrm{m}^{-1}$. Plus: $\mu_{\mathrm{i}} / \mu_{\mathrm{o}}=10.6, \gamma=0.26 \mathrm{mN} \mathrm{m}^{-1}$. Cross: $\mu_{\mathrm{i}} / \mu_{\circ}=25.2, \gamma=2.6 \mathrm{mN} \mathrm{m}^{-1}$. Diamond: $\mu_{\mathrm{i}} / \mu_{\circ}=44.7, \gamma=2.1 \mathrm{mN} \mathrm{m}^{-1}$. The background colors of blue and red for jetting and dripping respectively are added to aid visualization of the different regimes.

viscous forces of the inner and outer phase, exceeds the interfacial force. The dripping-to-jetting transition can be characterized by the relation:

$$
0.1<\frac{\sum F_{\mathrm{i}}}{F_{\mathrm{IFT}}}+\frac{\sum F_{\mathrm{o}}}{F_{\mathrm{IFT}}}<1
$$

confirming our hypothesis.

\section{Implications to interfacial tension estimation}

Our proposed characterization of dripping-to-jetting transition holds for a wide range of low interfacial tension and viscosity; hence it may be useful as a tool for estimating interfacial tension values..$^{34}$ Based on the balance of the downstream and upstream forces at the dripping-to-jetting transition, the interfacial tension can be estimated by the following (see part 5 in the ESI†):

$$
\gamma_{\text {estimated }} \sim 5\left(\rho_{\mathrm{i}} L_{\mathrm{i}} V_{\mathrm{i}, \mathrm{t}}^{2}+\mu_{\mathrm{i}} V_{\mathrm{i}, \mathrm{t}}+\mu_{\mathrm{o}} V_{\mathrm{o}, \mathrm{t}}\right)
$$

Over a wide range of relatively low interfacial tensions, the estimated values match well with those measured using a commercial spinning drop tensiometer (SITE100, KRÜSS), as shown in Fig. 4. These results provide further confirmation of our characterization of the dripping-to-jetting transition for an ATPS jet with low interfacial tension. 


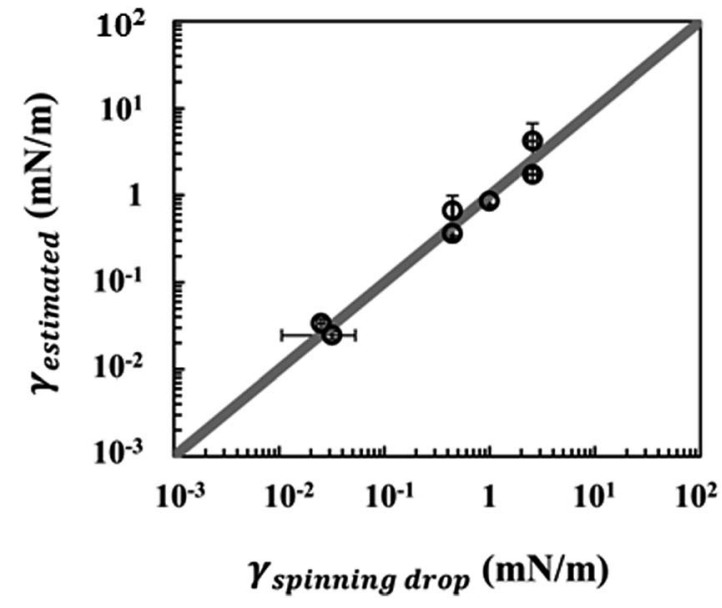

Fig. $4 \log -\log$ plot of $\gamma_{\text {estimated }}$ against $\gamma_{\text {spinning drop }}$ over a wide range of low interfacial tension values. $\gamma_{\text {estimated }}$ is the estimated value calculated by our proposed relation. $\gamma_{\text {spinning drop }}$ is the measured value obtained by a spinning drop tensiometer. The slope of grey line is unity.

\section{Conclusions}

In conclusion, our results highlight the importance of the critical jet velocity in the dripping-to-jetting transition of co-axial flow with low interfacial tension. The low jet velocity at the dripping-to-jetting transition indicates an exceptionally small inertial force and hence a relatively important viscous force for ATPSs with low interfacial tension $\left(\gamma<1 \mathrm{mN} \mathrm{m}^{-1}\right)$. The dripping-to-jetting transition is more properly characterized by the force balance between the interfacial force and the sum of downstream forces that does not neglect the viscous effect of the jet phase. Using this new characterization we also devise a method to estimate the low interfacial tension. By extending the understanding of the dripping-to-jetting transition to systems with low interfacial tension, our work lays a foundation to achieve control over formation of jets and drops in these systems.

\section{Acknowledgements}

We thank Dr Tiantian Kong, Dr Yuan Liu, Dr Zhou Liu and Prof. Howard A. Stone for valuable discussions. This research was supported by the Early Career Scheme (No. HKU 707712P), the General Research Fund (No. HKU 719813E, 17304514 and 17306315) and the Collaborative Research Fund (C6004-14G) from the Research Grants Council of Hong Kong, the General Program (No. 21476189/B060201) and the Major Research Plan (No. 91434202) from the National Natural Science Foundation of China, as well as the Seed Funding Programme for Basic Research (No. 201411159038, 201511159280) from the University of Hong Kong.

\section{References}

1 J. Eggers and E. Villermaux, Rep. Prog. Phys., 2008, 71, 036601 .
2 P. B. Umbanhowar, V. Prasad and D. A. Weitz, Langmuir, 2000, 16, 347-351.

3 P. Guillot, A. Colin and A. Ajdari, Phys. Rev. E: Stat., Nonlinear, Soft Matter Phys., 2008, 78, 016307.

4 S. Marre, C. Aymonier, P. Subra and E. Mignard, Appl. Phys. Lett., 2009, 95, 134105.

5 J. J. Kaufman, G. Tao, S. Shabahang, E.-H. Banaei, D. S. Deng, X. Liang, S. G. Johnson, Y. Fink and A. F. Abouraddy, Nature, 2012, 487, 463-467.

6 Y. K. Li, G. T. Liu, J. H. Xu, K. Wang and G. S. Luo, RSC Adv., 2015, 5, 27356-27364.

7 I. Ziemecka, V. van Steijn, G. J. M. Koper, M. T. Kreutzer and J. H. van Esch, Soft Matter, 2011, 7, 9878-9880.

8 A. Sauret and H. C. Shum, Appl. Phys. Lett., 2012, 100, 154106. 9 Y. Song and H. C. Shum, Langmuir, 2012, 28, 12054-12059. 10 S. Hardt and T. Hahn, Lab Chip, 2012, 12, 434-442.

11 Y. Song, Y. K. Chan, Q. Ma, Z. Liu and H. C. Shum, ACS Appl. Mater. Interfaces, 2015, 7, 13925-13933.

12 B.-U. Moon, S. G. Jones, D. K. Hwang and S. S. H. Tsai, Lab Chip, 2015, 15, 2437-2444.

13 H. C. Shum, J. Varnell and D. A. Weitz, Biomicrofluidics, 2012, 6, 012808.

14 A. Sauret, C. Spandagos and H. C. Shum, Lab Chip, 2012, 12, 3380-3386.

15 S. D. Geschiere, I. Ziemecka, V. van Steijn, G. J. M. Koper, J. H. V. Esch and M. T. Kreutzer, Biomicrofluidics, 2012, 6, 022007.

16 B.-U. Moon, N. Abbasi, S. G. Jones, D. K. Hwang and S. S. H. Tsai, Anal. Chem., 2016, 88, 3982-3989.

17 A. S. Utada, A. Fernandez-Nieves, H. A. Stone and D. A. Weitz, Phys. Rev. Lett., 2007, 99, 094502.

18 E. Castro-Hernández, V. Gundabala, A. Fernández-Nieves and J. M. Gordillo, New J. Phys., 2009, 11, 075021.

19 M. L. Cordero, F. Gallaire and C. N. Baroud, Phys. Fluids, 2011, 23, 094111.

20 J. K. Nunes, S. S. H. Tsai, J. Wan and H. A. Stone, J. Phys. D: Appl. Phys., 2013, 46, 114002.

21 Y. Ren, Z. Liu and H. C. Shum, Lab Chip, 2015, 15, 121-134.

22 A. S. Utada, A. Fernandez-Nieves, J. M. Gordillo and D. A. Weitz, Phys. Rev. Lett., 2008, 100, 014502.

23 A. S. Utada, L. Y. Chu, A. Fernandez-Nieves, D. R. Link, C. Holtze and D. A. Weitz, MRS Bull., 2007, 32, 702-708.

24 E. C. Bingham and R. F. Jackson, Standard substances for the calibration of viscometers, US Government Printing Office, 1917.

25 J. R. Lister and H. A. Stone, Phys. Fluids, 1998, 10, 2758-2764. 26 T. Cubaud and T. G. Mason, Phys. Fluids, 2008, 20, 053302. 27 Y. Song, A. Sauret and H. C. Shum, Biomicrofluidics, 2013, 7, 061301.

28 I. Ziemecka, V. van Steijn, G. J. M. Koper, M. Rosso, A. M. Brizard, J. H. van Esch and M. T. Kreutzer, Lab Chip, 2011, 11, 620-624.

29 J. Wu, T. Kong, K. W. K. Yeung, H. C. Shum, K. M. C. Cheung, L. Wang and M. K. T. To, Acta Biomater., 2013, 9, 7410-7419.

30 U. Shimanovich, Y. Song, J. Brujic, H. C. Shum and T. P. J. Knowles, Macromol. Biosci., 2015, 15, 501-508. 
31 Q. Ma, Y. Song, G. Baier, C. Holtze and H. C. Shum, J. Mater. Chem. B, 2016, 4, 1213-1218.

32 Y. Song, Z. Liu, T. Kong and H. Cheung Shum, Chem. Commun., 2013, 49, 1726-1728.

33 S. Y. Mak, Z. Li, A. Frere, T. C. Chan and H. C. Shum, Sci. Rep., 2014, 4, 6675.
34 J. H. Xu, S. W. Li, W. J. Lan and G. S. Luo, Langmuir, 2008, 24, 11287-11292.

35 C. C. de Oliveira, J. S. D. R. Coimbra, A. D. G. Zuniga, J. P. Martins and A. M. D. O. Siqueira, J. Chem. Eng. Data, 2012, 57, 1648-1652. 